(C) 2010 IEEE. Personal use of this material is permitted. Permission from IEEE must be obtained for all other uses, in any current or future media, including reprinting/republishing this material for advertising or promotional purposes, creating new collective works, for resale or redistribution to servers or lists, or reuse of any copyrighted component of this work in other works. 


\title{
SINR Degradation in MIMO-OFDM Systems with Channel Estimation Errors and Partial Phase Noise Compensation
}

\author{
Roberto Corvaja, Member, IEEE, and Ana García Armada, Senior Member, IEEE
}

\begin{abstract}
The phase noise effect in multiple-input-multipleoutput systems employing orthogonal frequency division multiplexing is considered in a realistic scenario where the estimated channel matrix is affected by an error. The analytical SINR degradation due to phase noise and channel estimation is obtained for linear receivers (ZF and MMSE).
\end{abstract}

Index Terms-MIMO-OFDM systems, phase noise, channel estimation, inter-channel interference, linear receivers.

\section{INTRODUCTION}

$\mathbf{O}$ RTHOGONAL Frequency Division Multiplexing (OFDM) is becoming the most frequently used technique for wireless systems, such as Long Term Evolution (LTE), and enhanced standards are contemplating its combination with multiple input-multiple output (MIMO). These systems suffer from inter-channel interference (ICI) introduced by phase noise and channel estimation errors. In most of the works these impairments are treated separately [1]-[4]: with ICI reduction schemes assuming perfect channel knowledge or analyzing the channel estimation error without phase noise. In [1] a scheme is proposed to remove the common phase error (CPE) and in [3] an iterative technique is presented to cancel successively the ICI terms, both assuming ideal channel estimation. In [2] the ICI power is obtained for zero-forcing (ZF) with ideal channel estimation by a frst-order approximation of the phase noise term. The channel estimation error is analyzed in [4] for $\mathrm{ZF}$, in fat fading, without phase noise. In [5]-[7] the effects are considered jointly for SISO-OFDM. The combination of MIMO with OFDM introduces the spatial dimension that causes additional interference. Depending on the chosen receiver scheme, it will have a different impact on the ICI. This is more accentuated if the channel is spatially correlated, as we will show. The degradation of phase noise and channel estimation for MIMO-OFDM is analyzed in [8] where the signal to interference plus noise ratio (SINR) is derived before the application of the receiver. However, phase noise and channel estimation errors are not separable and have different effects depending on the type of receiver. Therefore, it is important to consider them jointly and to characterize the degradation after the equalization of the receiver. Here we

R. Corvaja is with the Department of Information Engineering, University of Padova, Italy (e-mail: corvaja@dei.unipd it).

A. García Armada is with the Department of Signal Theory and Communications, University Carlos III of Madrid, Spain (e-mail: agarcia@tsc.uc3m.es).

This work is partly funded by the projects "COMONSENS" CSD200800010 and "MULTI-ADAPTIVE" TEC2008-06327-C03-02.
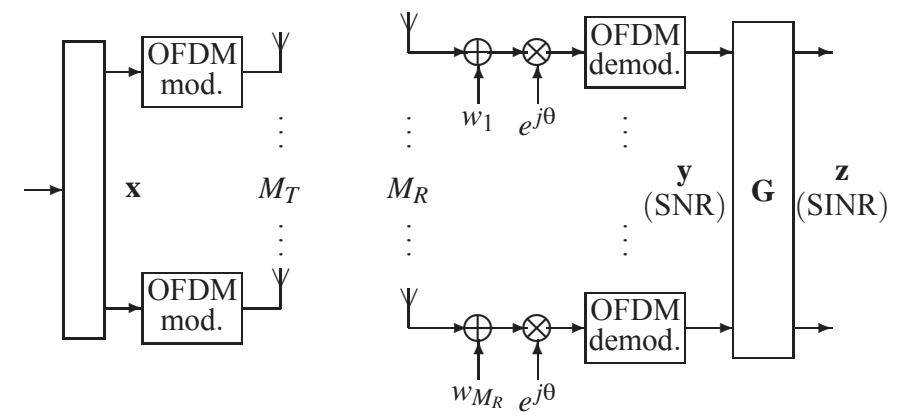

Fig. 1. Scheme of the MIMO-OFDM transmission system.

derive an analytical expression of the SINR degradation after two types of linear receivers, ZF and minimum mean squared error (MMSE). Moreover, we evaluate this degradation in the general case of spatially correlated multipath channel and without any approximation of the phase noise term. Some preliminary results were presented in [9], resorting mainly to simulations. Here we present in detail the analytical derivation of the SINR degradation. In [10] part of these analytical results was used to discuss the system parameters (number of antennas, multipath and phase noise conditions, and estimation errors) only for a spatially white channel.

\section{MIMO-OFDM SYSTEM}

The spatial multiplexing MIMO-OFDM system [11] is shown in Fig. 1, where $M_{T}$ independent data streams are OFDM modulated over $N$ sub-carriers and sent to $M_{T}$ transmit antennas. The receiver has $M_{R}$ antennas. The vector of transmitted symbols is $\mathbf{x}=\left[\mathbf{x}_{0}^{T}, \ldots, \mathbf{x}_{N-1}^{T}\right]^{T}$, where each component $\mathbf{x}_{n}=\left[x_{n, 1}, \ldots, x_{n, M_{T}}\right]^{T}$ groups the symbols transmitted on the $n$th sub-carrier on all the antennas. For each antenna pair $(i, j), i=1, \ldots, M_{R}, j=1, \ldots, M_{T}$ we have a multipath $M_{R} \times M_{T}$ impulse response $\mathbf{h}_{m}[i, j], m=0, \ldots, N_{C h}$, with length $N_{C h}$ shorter than the cyclic pref x. The elements of $\mathbf{h}_{m}$ are randomly distributed with powers determined according to the power delay profle. The spatial correlation is characterized by $E\left[\mathbf{H}_{n} \mathbf{H}_{n}^{H}\right]=\mathbf{R}_{R}, E\left[\mathbf{H}_{n}^{H} \mathbf{H}_{n}\right]=\mathbf{R}_{T}$ where $(\cdot)^{H}$ denotes the conjugate transpose. In a separable channel model, $\mathbf{R}_{T}$ and $\mathbf{R}_{R}$ correspond to the antenna correlations at transmitter and receiver, respectively. The phase noise $\theta(t)$ at the receiver, sampled at $k T, \theta_{k}=\theta(k T)$, coming mainly from the downconversion by high-frequency oscillators, is assumed to be the same for all the antennas. The received signal after the discrete Fourier transform (DFT), $\mathbf{y}=\left[\mathbf{y}_{0}^{T}, \ldots, \mathbf{y}_{N-1}^{T}\right]^{T}$, with $\mathbf{y}_{n}=\left[y_{n, 1}, \ldots, y_{n, M_{R}}\right]^{T}$ grouping all the signals on sub-carrier $n$, is

$$
\mathbf{y}=\mathbf{Q H x}+\mathbf{w} .
$$


$\mathbf{H}=\operatorname{diag}\left[\mathbf{H}_{0}, \mathbf{H}_{1}, \ldots, \mathbf{H}_{N-1}\right]$ is the $M_{R} N \times M_{T} N$ block diagonal channel frequency response, where each block is the $n$th subcarrier component of the channel DFT,

$$
\mathbf{H}_{n}=\sum_{m=0}^{N_{C h}-1} \mathbf{h}_{m} e^{-j 2 \pi \frac{m n}{N}} .
$$

The phase noise matrix $\mathbf{Q}$ in (1) is

$$
\mathbf{Q}=\left[\begin{array}{cccc}
\Theta_{0} & \Theta_{N-1} & \cdots & \Theta_{1} \\
\Theta_{1} & \Theta_{0} & \cdots & \Theta_{2} \\
\vdots & \vdots & \ddots & \vdots \\
\Theta_{N-1} & \Theta_{N-2} & \cdots & \Theta_{0}
\end{array}\right] \otimes \mathbf{I}_{M_{R}}
$$

where $\otimes$ is the Kronecker product and $\Theta_{n}$ is the $n$th component of the phase noise vector DFT

$$
\Theta_{n}=\sum_{k=0}^{N-1} e^{j \theta_{k}} e^{-j 2 \pi \frac{k n}{N}} .
$$

The value $E\left[\left|\Theta_{n}\right|^{2}\right]$, required for the ICI power, can be obtained by the phase noise spectral characteristics: $E\left[\left|\Theta_{n}\right|^{2}\right]$ is the power spectral density (PSD) of the sampled phase noise process $\mathcal{P}_{\theta}^{(s)}(f)$, evaluated at the $n$th sub-carrier frequency, $n \Delta f$, where $\Delta f=1 /(N T)$ is the sub-carrier spacing. $\mathcal{P}_{\theta}^{(s)}(f)$ is related to the continuous-time phase noise PSD $\mathcal{P}_{\theta}(f)$ by periodic repetition

$$
E\left[\left|\Theta_{n}\right|^{2}\right]=\mathcal{P}_{\theta}^{(s)}(n \Delta f)=\sum_{k=0}^{+\infty} \mathcal{P}_{\theta}(n \Delta f+k N \Delta f) .
$$

The phase noise PSD in PLL-based frequency synthesizers can be expressed as a weighted sum of components $\mathcal{P}_{\theta}(f)=$ $a_{0}+a_{1}\left(\frac{f_{1}}{f}\right)+a_{2}\left(\frac{f_{2}}{f^{2}}\right)+a_{3}\left(\frac{f_{3}}{f^{3}}\right)$, where the characteristic frequencies of each component $f_{i}$ and the relative weights $a_{i}$ strongly depend on the actual technology and device [12]. In the case of free running oscillators, they can be accurately characterized by a Wiener phase noise, which corresponds only to the $1 / f^{2}$ PSD component. In this case the amount of phase noise is usually expressed by the $3-\mathrm{dB}$ carrier bandwidth $B$, normalized to the sub-carrier spacing $B_{\theta}=B N T$. An alternative approach often employed is the approximation of the exponential $e^{j \theta_{k}}$ by its $\mathrm{frst}$ order Taylor series expansion, $1+j \theta_{k}$, which holds in the case of small phase noise [2].

Finally $\mathbf{w}$ in (1) is the AWGN contribution, where the phase noise is neglected, due to circular symmetry. We can then introduce an overall equivalent channel matrix $\mathbf{H}_{e q}=\mathbf{Q H}$, giving $\mathbf{y}=\mathbf{H}_{e q} \mathbf{x}+\mathbf{w}$. We def ne the reference signal to noise ratio (SNR) as the ratio between the useful component and the noise power, $S N R=E\left[\left(\mathbf{H}_{e q} \mathbf{x}\right)^{H}\left(\mathbf{H}_{e q} \mathbf{x}\right)\right] / E\left[\mathbf{w}^{H} \mathbf{w}\right]$.

\section{RECEIVER ScheMES}

We will analyze linear receivers (ZF and MMSE), where the recovered signal is obtained by $\mathbf{Z}=\mathbf{G y}$. With $Z F$ the matrix $\mathbf{G}_{Z F}$, which removes the spatial interference at the expense of enhancing the additive noise, is $\mathbf{G}_{Z F}=\tilde{\mathbf{H}}_{e q}^{\dagger}$, where $(\cdot)^{\dagger}$ denotes the Moore-Penrose pseudo-inverse and $\tilde{\mathbf{H}}_{e q}=\tilde{\mathbf{H}} \tilde{\mathbf{Q}}$ is the estimated overall channel matrix. With MMSE the matrix $\mathbf{G}_{M M S E}$, balancing the spatial interference with the noise, is

$$
\mathbf{G}_{M M S E}=\left(\tilde{\mathbf{H}}_{e q}^{H} \tilde{\mathbf{H}}_{e q}+\frac{1}{S N R} \mathbf{I}_{N M_{T}}\right)^{-1} \tilde{\mathbf{H}}_{e q}^{H} .
$$

For both receivers we assume that $\mathrm{f}$ rst the $\mathrm{CPE}$ is compensated [1] by multiplication of the received signal by the matrix $\tilde{\Theta}_{0}^{-1} \mathbf{I}_{N M_{R}}$, in other words $\mathbf{Q}$ is approximated by its diagonal elements $\tilde{\mathbf{Q}}=\tilde{\Theta}_{0} \mathbf{I}_{N M_{R}}$ and $\tilde{\mathbf{H}}_{e q}=\tilde{\Theta}_{0} \tilde{\mathbf{H}}$. The CPE can be estimated by means of $N_{p}$ pilot sub-carriers, inserted in the OFDM symbol at positions $p_{i}, i=1, \ldots, N_{p}$ on the antenna streams $M_{j}, j=1, \ldots, M_{p}$. It is estimated as the mean phase displacement with respect to the expected symbol [13], by an average

$$
\tilde{\Theta}_{0}=\frac{1}{M_{p}} \frac{1}{N_{p}} \sum_{i=1}^{N_{p}} \sum_{j=1}^{M_{p}} \frac{y_{p_{i}, M_{j}}}{x_{p_{i}, M_{j}}\left|y_{p_{i}, M_{j}}\right|},
$$

where $y_{p_{i}, M_{j}}$ denotes the received value at the positions where the pilot symbols are placed, and $x_{p_{i}, M_{j}}$ is the corresponding pilot symbol value. The effect of the estimation process on $\Theta_{0}$ can be expressed by $\tilde{\Theta}_{0}=\Theta_{0}+\varepsilon_{C P E}$, where $\varepsilon_{C P E}$ is the error in the CPE estimate, with zero mean and variance $\sigma_{C P E}^{2}$. The residual error left by CPE estimation will depend on the number of pilots and phase noise characteristics. For example, with the CPE estimation technique reported in [16], 4 pilots out of 64 are enough to obtain the same performance as without phase noise when Wiener phase noise has $B_{\theta}<5 \cdot 10^{-2}$ and $S N R=30 \mathrm{~dB}$. Channel estimation in MIMO-OFDM uses typically pilot symbols scattered in time and frequency, and has been extensively analyzed (see for example [14] and references therein). Here, we include the f nal estimation error on $\mathbf{H}$ by an additive term $\mathbf{Z}$, and the actual channel matrix is $\mathbf{H}=\tilde{\mathbf{H}}+\mathbf{Z}$ where $\mathbf{Z}$ is independent of $\mathbf{H}$, with zeromean independent Gaussian elements $z[i, j]$ whose variance $\sigma_{e s t}^{2}$ is equal to the mean-squared error (MSE) obtained by the channel estimator. The accuracy of the channel estimate obtained by $N_{p}$ pilots inserted in the OFDM signal depends on the position of the pilots. The best achievable MSE for each SISO-OFDM channel is [17] $M S E=\left(\sigma_{n}^{2} N_{p}\right) / E_{p}$ where $E_{p}$ is the pilot energy and $\sigma_{n}^{2}$ the AWGN variance. Note that CPE and channel estimation are usually performed separately; moreover, from (7) the effect of a channel estimation error on $\mathbf{H}$ has a reduced effect on the CPE estimation, so that we consider $\mathbf{Z}$ and $\varepsilon_{C P E}$ independent.

\section{SINR DEGRADATION}

We def ne the SINR after the receiver as the ratio between the useful signal power $\sigma_{x}^{2}$ and the variance of the overall disturbance caused by noise and spatial interference $\sigma_{0}^{2}$, that is, $\operatorname{SINR}=\sigma_{x}^{2} / \sigma_{0}^{2}$. In ideal conditions, that is, without phase noise or estimation error, the SINR for the the $n$th signalling vector at the output of the $\mathrm{ZF}$ receiver is [15]

$$
\operatorname{SINR}_{n}=\frac{S N R / M_{T}}{\left[\mathbf{H}_{e q}^{H} \mathbf{H}\right]_{n, n}^{-1}}
$$

while for the MMSE receiver, on the $n$th signalling vector, it has the form

$$
\operatorname{SINR}_{n}=\frac{1}{\left[\left(S N R / M_{T}\right) \mathbf{H}_{e q}^{H} \mathbf{H}+\mathbf{I}_{N M_{T}}\right]_{n, n}^{-1}}-1
$$

where $[\cdot]_{n, n}$ indicates the $(n, n)$ th entry of a matrix. In the following evaluations the average SINR over all the signalling vectors is considered, $\mathrm{SINR}=\frac{1}{M_{T}} \sum_{n=1}^{M_{T}} \mathrm{SINR}_{n}$. 
The joint effects of phase noise and estimation error will be accounted for by the SINR degradation, def ned as the increase to the SINR giving the same error probability as in the ideal case of perfect channel knowledge and no phase noise. In the non-ideal case we should reduce the disturbance power by the overall interference power $\sigma_{I C I}^{2}$ to get the same performance, where

$$
\sigma_{I C I}^{2}=\frac{1}{N} \sum_{n=0}^{N-1} \sigma_{v_{n}}^{2}
$$

with $\sigma_{v_{n}}^{2}$ denoting the ICI variance on the $n$th sub-carrier, averaged over the $M_{T}$ transmit antennas, obtained in the next section. Then the degradation $D$ is the ratio between the new SINR, with disturbance power reduced by the ICI variance $\sigma_{I C I}^{2}$, and the original SINR, in $\mathrm{dB}$

$$
D=10 \log _{10} \frac{\sigma_{x}^{2} /\left(\sigma_{0}^{2}-\sigma_{I C I}^{2}\right)}{\sigma_{x}^{2} / \sigma_{0}^{2}}=10 \log _{10}\left(\frac{\sigma_{0}^{2}}{\sigma_{0}^{2}-\sigma_{I C I}^{2}}\right) .
$$

Clearly, from (11), the degradation goes to inf nite if $\sigma_{I C I}^{2}$ gets close to $\sigma_{0}^{2}$. This is a f oor effect due to the dominance of phase noise and channel imperfections at high SNR.

\section{Derivation of the ICI VARIANCE}

The overall interference term $\mathbf{v}$ at the decision point, which includes ICI and imperfect CPE estimation after equalization, is

$$
\mathbf{v}=\mathbf{G}\left[\left(\mathbf{Q}-\tilde{\Theta}_{0} \mathbf{I}_{N}\right) \otimes \mathbf{I}_{M_{R}}\right] \mathbf{H} \mathbf{x} .
$$

If we consider the component on the $n$th sub-carrier, we have

$$
\mathbf{v}_{n}=\mathbf{G}_{n} \sum_{i=0}^{N-1} \Theta_{i \neq n} \Theta_{n-i} \mathbf{H}_{i} \mathbf{x}_{i}+\varepsilon_{C P E} \mathbf{G}_{n} \mathbf{H}_{i} \mathbf{x}_{i}
$$

where $\mathbf{G}_{n}$ is the $n$th diagonal block of $\mathbf{G}$. The ICI variance on the $n$th sub-carrier, averaged over the transmit antennas $M_{T}$, is then

$$
\begin{aligned}
\sigma_{v_{n}}^{2} & =\frac{\sigma_{x}^{2}}{M_{T}} \sum_{i=0}^{N-1} E\left[\left|\Theta_{n-i}\right|^{2}\right] \operatorname{Tr}\left\{E\left[\mathbf{H}_{i}^{H} \mathbf{G}_{n}^{H} \mathbf{G}_{n} \mathbf{H}_{i}\right]\right\} \\
& +\sigma_{x}^{2} \frac{\sigma_{C P E}^{2}}{M_{T}} \operatorname{Tr}\left\{E\left[\mathbf{H}_{n}^{H} \mathbf{G}_{n}^{H} \mathbf{G}_{n} \mathbf{H}_{n}\right]\right\}
\end{aligned}
$$

where the symbols $\mathbf{x}_{i}$ have been assumed independent with $E\left[\mathbf{x}_{i} \mathbf{x}_{i}^{H}\right]=\sigma_{x}^{2} \mathbf{I}_{M_{T}}$.

\section{A. $Z F$ receiver}

In the expression of the ICI variance (14), for the ZF receiver we have

$$
\begin{aligned}
& \operatorname{Tr}\left\{E\left[\mathbf{H}_{i}^{H} \mathbf{G}_{n}^{H} \mathbf{G}_{n} \mathbf{H}_{i}\right]\right\}=\operatorname{Tr}\left\{E\left[\mathbf{H}_{i}^{H}\left(\mathbf{H}_{n}^{\dagger}\right)^{H} \mathbf{H}_{n}^{\dagger} \mathbf{H}_{i}\right]\right\} \\
& +\operatorname{Tr}\left\{E\left[\mathbf{Z}_{i}^{H}\left(\mathbf{H}_{n}^{\dagger}\right)^{H} \mathbf{H}_{n}^{\dagger} \mathbf{Z}_{i}\right]\right\} .
\end{aligned}
$$

Because of the independence of $\mathbf{Z}$ and $\mathbf{H}$ and $E\left[\mathbf{Z}_{i} \mathbf{Z}_{i}^{H}\right]=$ $\sigma_{e s t}^{2} \mathbf{I}$, we have

$$
\begin{aligned}
& \operatorname{Tr}\left\{E\left[\mathbf{Z}_{i}^{H}\left(\mathbf{H}_{n}^{\dagger}\right)^{H} \mathbf{H}_{n}^{\dagger} \mathbf{Z}_{i}\right]\right\} \\
& =\sigma_{e s t}^{2} \operatorname{Tr}\left\{E\left[\left(\mathbf{H}_{n}^{H} \mathbf{H}_{n}\right)^{-1}\right]\right\}=\sigma_{\text {est }}^{2} \frac{\operatorname{Tr}\left[\mathbf{R}_{T}^{-1}\right]}{M_{R}-M_{T}},
\end{aligned}
$$

for $M_{R}>M_{T}$, since the above term represents the expected value of the trace of an inverse Wishart matrix [18]. To evaluate the f rst right-hand-side term of (15), the joint channel frequency response statistics on sub-carriers $i$ and $n$ are required. The channel frequency response on different subcarriers can be expressed by a combination of a totally correlated component and an independent component, weighted by the sub-carrier correlation $\rho$, namely $\mathbf{H}_{i}=\rho \mathbf{H}_{n}+\sqrt{1-\rho} \mathbf{H}_{I}$, where $\mathbf{H}_{I}$ is independent of $\mathbf{H}_{n}$, and the correlation is the Fourier transform of the power delay prof le (PDP) evaluated at $(n-i) \Delta f$

$$
\rho=E\left[\mathbf{H}_{i}(j, k) \mathbf{H}_{n}(j, k)\right]=\sum_{m=1}^{N_{c H}} E\left[\left|\mathbf{h}_{m}(j, k)\right|^{2} e^{j 2 \pi m(n-i) \Delta f} .\right.
$$

For example, for an exponential PDP, $\rho=\frac{1}{1+j 2 \pi(n-i) \Delta f T_{r m s}}$, where $T_{r m s}$ is the channel r.m.s. delay spread. Here we assume that the PDP does not depend on the transmit-receive antenna pair, as in [19]. The case of different PDP for different antenna pairs is detailed in the Appendix. Considering the f rst (correlated) component, $\rho \mathbf{H}_{n}$, we have

$$
\operatorname{Tr}\left\{E\left[\left(\mathbf{H}_{n}^{\dagger} \mathbf{H}_{n}\right)^{H} \mathbf{H}_{n}^{\dagger} \mathbf{H}_{n}\right]\right\}=M_{T} .
$$

Considering the second component, $\sqrt{1-|\rho|^{2}} \mathbf{H}_{I}$, by applying the trace property $\operatorname{Tr}[A B]=\operatorname{Tr}[B A]$ and the independence between $\mathbf{H}_{n}$ and $\mathbf{H}_{I}$, we have

$$
\begin{aligned}
\operatorname{Tr}\left\{E\left[\mathbf{H}_{I}^{H}\left(\mathbf{H}_{n}^{\dagger}\right)^{H} \mathbf{H}_{n}^{\dagger} \mathbf{H}_{I}\right]\right\} & =\operatorname{Tr}\left\{E\left[\left(\mathbf{H}_{n}^{H} \mathbf{H}_{n}\right)^{\dagger}\right] E\left[\mathbf{H}_{I} \mathbf{H}_{I}^{H}\right]\right\} \\
& =M_{R} .
\end{aligned}
$$

For the particular case of Wiener phase noise and spatially incorrelated channel with exponential PDP, the variance of the overall phase noise interference after ZF can be summarized in

$$
\begin{aligned}
\sigma_{v_{n}}^{2}= & \sigma_{x}^{2} \sigma_{C P E}^{2}+\frac{\sigma_{x}^{2}}{M_{T}} \sum_{\substack{i=0 \\
i \neq n}}^{N-1} \sum_{k=0}^{+\infty} \frac{2 \pi B_{\theta}}{1+\left(\frac{(n-i) / N+k}{B_{\theta}}\right)^{2}} \\
\times & {\left[\frac{\sigma_{e s t}^{2} M_{T}}{M_{R}-M_{T}}+\frac{M_{T}}{1+4 \pi^{2}(n-i)^{2}\left(\Delta f T_{r m s}\right)^{2}}\right.} \\
& \left.+\frac{4 \pi^{2}(n-i)^{2}\left(\Delta f T_{r m s}\right)^{2}}{1+4 \pi^{2}(n-i)^{2}\left(\Delta f T_{r m s}\right)^{2}} M_{T} M_{R}\right]
\end{aligned}
$$

\section{B. MMSE receiver}

Also in the case of the MMSE receiver, in the trace of the inner matrix of (14), we can separate the effects of the estimation error and of the sub-carrier correlation,

$$
\begin{aligned}
& \operatorname{Tr}\left\{E\left[\mathbf{H}_{i}^{H} \mathbf{G}_{n}^{H} \mathbf{G}_{n} \mathbf{H}_{i}\right]\right\}=\sigma_{e s t}^{2} E\left[\operatorname{Tr}\left\{\mathbf{G}_{n}^{H} \mathbf{G}_{n}\right\}\right] \\
& +\operatorname{Tr}\left\{E \left[\mathbf{H}_{i}^{H} \mathbf{H}_{n}\left\{\left(\mathbf{H}_{n}^{H} \mathbf{H}_{n}+\frac{1}{S N R} \mathbf{I}_{M_{T}}\right)^{-1}\right\}^{H}\right.\right. \\
& \left.\left.\times\left(\mathbf{H}_{n}^{H} \mathbf{H}_{n}+\frac{1}{S N R} \mathbf{I}_{M_{T}}\right)^{-1} \mathbf{H}_{n}^{H} \mathbf{H}_{i}\right]\right\} .
\end{aligned}
$$

Again we can express $\mathbf{H}_{i}$ as $\mathbf{H}_{i}=\rho \mathbf{H}_{n}+\sqrt{1-\rho} \mathbf{H}_{I}$, where the latter is independent of $\mathbf{H}_{n}$. The difference between the MMSE and ZF is relevant in the low SNR region, since for 


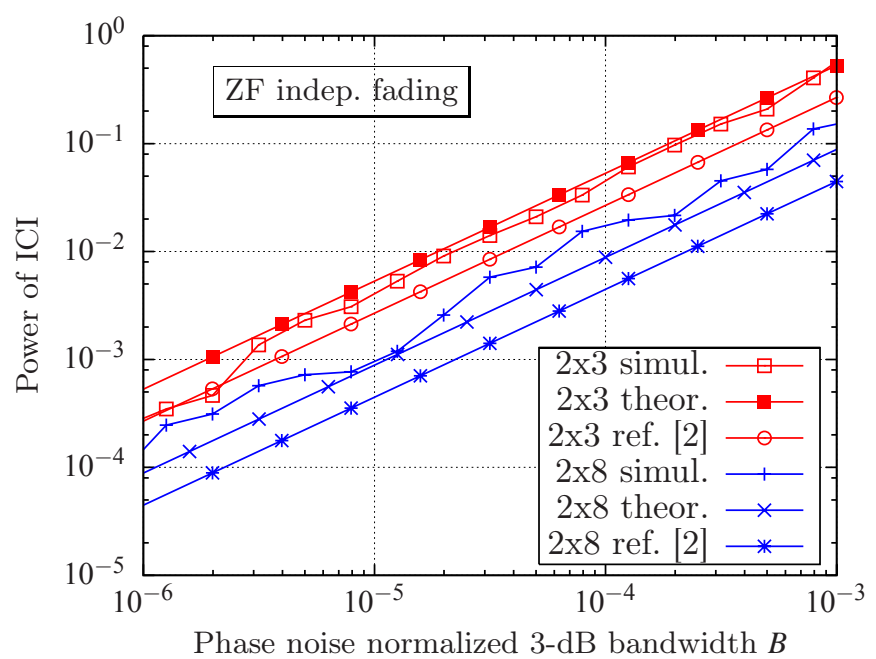

Fig. 2. Power of the ICI contribution for the ZF receiver with independent fading and ideal channel estimation.

high SNR $\mathbf{G}_{M M S E}$ converges to $\mathbf{G}_{Z F}$. Then, for low SNR, $\mathbf{G}_{n}$ can be approximated as $\mathbf{G}_{n}=S N R \mathbf{H}_{n}^{H}$, and

$$
E\left[\operatorname{Tr}\left\{\mathbf{G}_{n}^{H} \mathbf{G}_{n}\right\}\right]=S N R^{2} E\left[\operatorname{Tr}\left\{\mathbf{H}_{n}^{H} \mathbf{H}_{n}\right\}\right]=S N R^{2} M_{R} \operatorname{Tr}\left[\mathbf{R}_{T}\right] .
$$

In fact the expectation in (22) represents the mean value of the trace of a Wishart matrix [18]. For the correlated component, the expected trace of the square of a Wishart matrix [18] gives

$$
\operatorname{Tr}\left\{E\left[\mathbf{H}_{n} \mathbf{H}_{n}^{H} \mathbf{H}_{n} \mathbf{H}_{n}^{H}\right]\right\}=M_{T} M_{R}\left[\operatorname{Tr}\left(\mathbf{R}_{R}^{2}\right)+\frac{1}{M_{T}} \operatorname{Tr}^{2}\left(\mathbf{R}_{R}\right)\right] .
$$

In the independent fading term, we have the expected trace of the product of independent matrices [18] with $\operatorname{Tr}\left\{E\left[\mathbf{H}_{n} \mathbf{H}_{n}^{H} \mathbf{H}_{I} \mathbf{H}_{I}^{H}\right]\right\}=M_{T} \operatorname{Tr}\left[\mathbf{R}_{R}^{2}\right]$.

With MMSE at low SNR, the overall phase noise interference variance for the particular case of Wiener phase noise and spatially incorrelated channel with exponential PDP, is

$$
\begin{aligned}
\sigma_{v_{n}}^{2}= & \sigma_{x}^{2} \sigma_{C P E}^{2}+\frac{\sigma_{x}^{2}}{M_{T}} \sum_{\substack{i=0 \\
i \neq n}}^{N-1} \sum_{k=0}^{+\infty} \frac{2 \pi B_{\theta}}{1+\left(\frac{(n-i) / N+k}{B_{\theta}}\right)^{2}} \\
\times & {\left[\frac{\sigma_{e s t}^{2} M_{T}}{M_{R}-M_{T}}+\frac{S N R^{2} M_{T} M_{R}\left(M_{T}+M_{R}\right)}{1+4 \pi^{2}(n-i)^{2}\left(\Delta f T_{r m s}\right)^{2}}\right.} \\
& \left.+\frac{4 \pi^{2}(n-i)^{2}\left(\Delta f T_{r m s}\right)^{2}}{1+4 \pi^{2}(n-i)^{2}\left(\Delta f T_{r m s}\right)^{2}} S N R^{2} M_{T}^{2} M_{R}\right]
\end{aligned}
$$

\section{Performance Results}

To validate the above expressions, we show some results with $N=64$ sub-carriers and, without loss of generality, the same variance of the estimation error on the channel and on the $\mathrm{CPE}, \sigma_{e s t}^{2}=\sigma_{C P E}^{2}$. First, we present the case of independent fading among the sub-carriers and Wiener phase noise, to compare our analytic approach with simulations and with [2]. The curves of the ICI power vs. $B_{\theta}$ of Fig. 2 show a very good matching, although the frst order approximation of [2] is slightly optimistic. Note that the assumption of independent fading in sub-carriers is an idealization analyzed here just to compare with [2]. In practical systems, pilot-based channel estimation would not work in such circumstances so that the

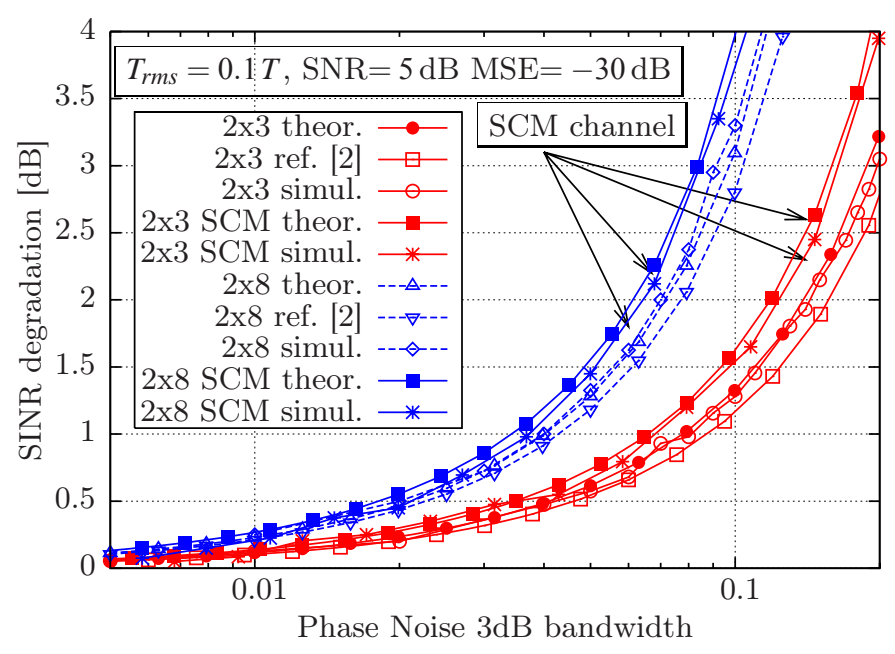

Fig. 3. Degradation for $\mathrm{ZF}$ as a function of the phase noise bandwidth, with exponential PDP $T_{r m s}=0.1 T$ or SCM channel, estimation error MSE= $-30 \mathrm{~dB}$ and reference $\mathrm{SNR}=5 \mathrm{~dB}$.

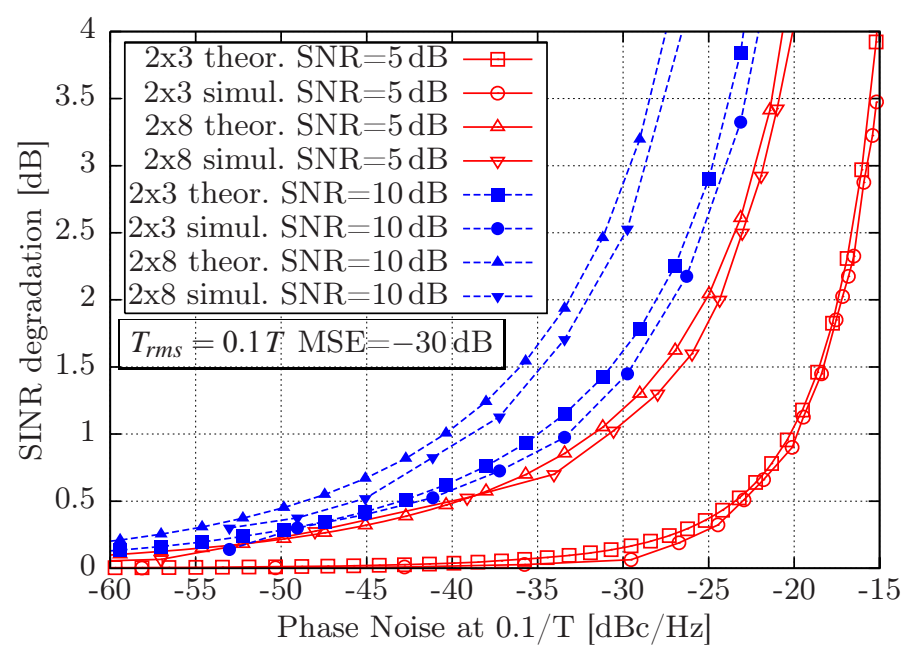

Fig. 4. Degradation for MMSE as a function of the phase noise PSD at $0.1 / T$, with exponential PDP $T_{r m s}=0.1 T$, estimation error $\mathrm{MSE}=-30 \mathrm{~dB}$ and reference $\mathrm{SNR}=5 \mathrm{~dB}$ and $\mathrm{SNR}=10 \mathrm{~dB}$.

number of sub-carriers is always properly designed to avoid this condition. As examples of more realistic channel models, we use a spatially uncorrelated channel with exponential PDP with $T_{r m s}=0.1$, and on the other hand also a fully realistic spatially correlated channel with the spatial correlation and the PDP specifed by 3GPP for the LTE evaluation [19]. Fig. 3 shows the SINR degradation of $\mathrm{ZF}$ for $\mathrm{SNR}=5 \mathrm{~dB}$ and estimation error $\mathrm{MSE}=-30 \mathrm{~dB}$, with a good $\mathrm{ft}$ between the simulations and the theoretical analysis and again [2] is slightly optimistic. To illustrate the performance of MMSE, a more general phase noise model is adopted [12], with an example of PSD with $1 / f^{3}$ decay up to $f_{3}$, then $1 / f^{2}$ up to $f_{2}=10 f_{3}$, followed by a fxed white noise level of $-140 \mathrm{dBc} / \mathrm{Hz}$ for $f>f_{2}$. The value in $\mathrm{dBc} / \mathrm{Hz}$ of the phase noise PSD at $0.1 \Delta f$ is considered as a parameter, thus setting $f_{3}$. Fig. 4 compares the theoretical SINR degradation with the simulation values, again with $\mathrm{MSE}=-30 \mathrm{~dB}$, for reference $\mathrm{SNR}=5 \mathrm{~dB}$ and $10 \mathrm{~dB}$. We note a very good match 
between the theoretical and the simulation results, especially for low SNR, while at higher SNR values the theoretical result becomes an upper-bound.

\section{CONCLUSIONS}

We derived the expression of the SINR degradation in linear receivers for MIMO-OFDM, considering both the channel estimation error and the phase noise, with partial CPE compensation. The accuracy of the expressions has been compared to previously published works and verif ed with simulation results.

\section{APPENDIX}

\section{Channels with Different Power Delay Profiles}

In the case of different PDP describing the second-order distribution of the channel paths for different antenna pairs, we can use a decomposition similar to $\mathbf{H}_{i}=\rho \mathbf{H}_{n}+\sqrt{1-\rho} \mathbf{H}_{I}$, where instead of a scalar $\rho$ for all the matrix elements, we have different correlations, so that

$$
\mathbf{H}_{i}=\boldsymbol{\rho} \circ \mathbf{H}_{n}+\rho_{C} \circ \mathbf{H}_{I}
$$

where $\circ$ represents the Hadamard product, $\rho_{C}(i, j)=$ $\sqrt{1-|\boldsymbol{\rho}(i, j)|}$ and the elements of the matrix $\boldsymbol{\rho}$ are given by (17). In this case, when considering the expected trace of products of the kind $E\left[\rho \circ \mathbf{H}_{n}\left(\left(\boldsymbol{\rho} \circ \mathbf{H}_{n}\right)^{H}\right]\right.$, such as for example in (19) or (22) (similarly for $\rho_{C} \circ \mathbf{H}_{I}$ ), these can be seen as the expected value of the squared Frobenius norm of a Hadamard matrix product. Thus, we can derive an upper bound to the variance, by the property [20], $\|\mathbf{A} \circ \mathbf{A}\|_{2} \leq\|\mathbf{A}\|_{2}\|\mathbf{B}\|_{2}$. In practice the results (20) and (24), with $\|\rho\|_{2}^{2}$ and $\left\|\rho_{C}\right\|_{2}^{2}$ instead of $|\rho|^{2}$ and $\left(1-|\rho|^{2}\right)$, would represent an upper bound to the variance of the overall interference.

\section{REFERENCES}

[1] P. Liu, S. Songping, and Y. Bar-Ness, "A phase noise mitigation scheme for MIMO WLANs with spatially correlated and imperfectly estimated channels," IEEE Commun. Lett., vol. 10, no. 3, pp. 141-143, Mar. 2006.

[2] T. C. W. Schenk, X.-J. Tao, P. F. M. Smulders, and E. R. Fledderus, "On the inf uence of phase noise induced ICI in MIMO OFDM systems," IEEE Commun. Lett., vol. 9, no. 8, pp. 682-684, Aug. 2005.

[3] S. Bittner, E. Zimmermann, and G. Fettweis, "Iterative phase noise mitigation in MIMO-OFDM systems with pilot aided channel estimation," in Proc. IEEE VTC 2007 Fall, pp. 1087-1091, Sep. 2007.
[4] Cheng Wang, E. K. S. Au, R. D. Murch, W. H. Mow, R. S. Cheng, and $\mathrm{V}$. Lau, "On the performance of the MIMO zero-forcing receiver in the presence of channel estimation error," IEEE Trans. Wireless Commun., vol. 6, no. 3, pp. 805-810, Mar. 2007.

[5] F. Munier, T. Eriksson, and A. Svensson, "An ICI reduction scheme for OFDM system with phase noise over fading channels," IEEE Trans. Commun., vol. 56, no. 7, pp. 1119-1126, July 2008.

[6] F. Septier, Y. Delignon, A. Menhaj-Rivenq, and C. Garnier, "Monte Carlo methods for channel, phase noise, and frequency offset estimation with unknown noise variances in OFDM systems," IEEE Trans. Signal Process., vol. 56, no. 8, part 1, pp. 3613-3626, Aug. 2008.

[7] Q. Zou, A. Tarighat, and A. H. Sayed, "Compensation of phase noise in OFDM wireless systems," IEEE Trans. Signal Process., vol. 55, no. 11, pp. 5407-5424, Nov. 2007.

[8] Y. Zhang and H. Liu, "MIMO-OFDM systems in the presence of phase noise and doubly selective fading," IEEE Trans. Veh. Technol., vol. 56, no. 4, part 2, pp. 2277-2285, July 2007.

[9] R. Corvaja and A. García Armada, "Effect of channel estimation errors in MIMO-OFDM systems with phase noise compensation," in Proc. IEEE Globecom 2008, pp. 4599-4603, New Orleans, Nov. 2008.

[10] R. Corvaja and A. García Armada, "Effect of multipath and antenna diversity in MIMO-OFDM systems with imperfect channel estimation and phase noise compensation," Elsevier Physical Commun., vol. 1, no. 4, pp. 288-297, Dec. 2008.

[11] G. G. Raleigh and J. M. Cioff, "Spatio-temporal coding for wireless communication," IEEE Trans. Commun., vol. 46, no. 3, pp. 357-366, Mar. 1998.

[12] V. F. Kroupa, "Noise properties of PLL systems," IEEE Trans. Commun., vol. 30, no. 10, pp. 2244-2252, Oct. 1982.

[13] Y. Mostof and D. C. Cox, "ICI mitigation for pilot-aided OFDM mobile systems," IEEE Trans. Wireless Commun., vol. 4, no. 2, pp. 765-774, Mar. 2005.

[14] Z. Zhang, W. Zhang, and C. Tellambura, "MIMO-OFDM channel estimation in the presence of frequency offsets," IEEE Trans. Wireless Commun., vol. 7, no. 6, pp. 2329-2339, June 2008.

[15] A. Paulraj, Introduction to Space-Time Wireless Communications. Cambridge University Press, 2003.

[16] S. Wu and Y. Bar-Ness, "A phase noise suppression algorithm for OFDM-based WLANs," IEEE Commun. Lett., vol. 6, no. 12, pp. 535537, Dec. 2002.

[17] R. Neggi and J. M. Cioff, "Pilot tone selection for channel estimation in a mobile OFDM system," IEEE Trans. Consumer Electron., vol. 44, no. 3, pp. 1122-1128, Aug. 1998.

[18] D. Maiwald and D. Kraus, "Calculation of moments of complex Wishart and complex inverse Wishart distributed matrices," in IEE Proc. Radar, Sonar Navigation, vol. 147, no. 4, pp. 162-168, Aug. 2000.

[19] J. Salo, G. Del Galdo, J. Salmi, P. Kyösti, M. Milojevic, D. Laselva, and C. Schneider, "MATLAB implementation of the 3GPP spatial channel model (3GPP TR 25.996)." [Online]. Available: http://www.tkk.f/Units/ Radio/scm/, Jan. 2005.

[20] R. A. Horn, "The Hadamard product," in Proc. Sympos. Appl. Math., Amer. Math. Soc. vol. 40, pp. 87-169, 1990. 